\title{
Why a universe as a whole must be a quantum object
}

\author{
Pedro F. González-Díaz
}

Colina de los Chopos, Instituto de Física Fundamental, CSIC, Madrid, Spain; p.gonzalezdiaz@iff.csic.es

Received 3 March 2011; revised 21 March 2011; accepted 26 March 2011.

\begin{abstract}
Technical and physical reasons are given in favor of the idea that single universes, accelerating or not, essentially are quantum-mechanical entities without any classical analogs, even when they are forming part of a given multiverse.
\end{abstract}

Keywords: Decoherence; Baby Universes; Bell's Inequalities

\section{INTRODUCTION}

Why can't you be in two places at the same time? The simple, obvious answer is: because of your interaction with your environment, the superposition of states that you are actually quantum-mechanically collapses into a single classical state, what makes your personal and unique yourself a phenomenon called quantum decoherence. The ultimate reason for that phenomenon being operative for macroscopic objects and not with atoms and small molecules is still a mystery. Some have even thought that it is due to interaction with the gravitational waves that are pervading the whole universe. Whatever it could be the reason for decoherence in macroscopic object, what is very clear is that any universe as a whole has no environment at all and therefore no quantum decoherence may collapse its quantum state into a single classical one. Thus, the universe as a whole should be a real quantum object which is a superposition of states and cannot have any classical analog. It might still be argued that quantum entanglement between distinct universes or tunnelling strips like wormholes, ring holes or Klein-bottle holes connecting these universes to each other could well make the job of quantum decoherence rendering every individual single universe finally a classical object. However, the non locality properties of quantum entanglement among different universes are far from being shared by the required quantum decoherence which rather plays the quite more conventional classical role of the wave function collapse which can even be induced by gravitational interaction (actually in this case, besides the e.g. Zurek formulas [1], one should necessarily make recourse to that gravitational interaction which comes about due to correlated baby universe pairs [2] to make it compatible with the whole scenario we were dealing with) that might be a purely classical interaction at the end of the day, and the kind of tunnelling we have just mentioned are defined as being classical communication channels, at least when regarded as solutions to Einstein equations.

Thus, the main aim of the present report is at discussing several compelling arguments in favour of the idea that the single universe considered as a whole is purely a quantum object devoid of any classical analog. These arguments are based on the physics of baby universes which are branched off from a large parent universe in self entangled pairs using the method of quantum optical gravity [2], both under the Zurek perspective (Section 2) or employing field theory tools like the T-symmetry (Section 3). The problem of the placement of observers in quantum cosmology is briefly commented in Section 4 , and we conclude and add some extra comments in Section 5 .

\section{THE MASTER EQUATION}

In this and the next sections we shall use the quantum-optical formalism for quantum gravity [2] as applied to the density matrix of baby universes and scalar fields. We will restrict ourselves to the simplest case of the interaction between a massless, conformally invariant scalar matter field, $\Phi(x, t)$, and single doubly connected Euclidean wormholes [3], using the quantum-optical formalism of the density matrix in the interaction representation and ordinary time-dependent perturbation theory in first-order approximation. We consider the interaction Hamiltonian $H^{I}=\sum_{i} H_{i}^{I}(\Phi) A_{i}$, with $A_{i}$ the baby universe operators, and assume a full density matrix $\boldsymbol{\rho}=\boldsymbol{\rho}_{\Phi} \otimes \boldsymbol{\rho}_{b}=\boldsymbol{\rho}_{\Phi} \sum_{1, j}|i\rangle\langle j|$, where $\boldsymbol{\rho}_{\Phi}$ and $\boldsymbol{\rho}_{b}$ are density matrices for the scalar matter field and the baby universes, respectively. Now, from the Heisenberg equation of motion for the full density ma- 
trix, which we iterate for small increments of time, we obtain with the same approximation as used in ordinary time-dependent perturbation theory

$$
\dot{\rho}_{\Phi}(k, t)=\operatorname{Tr}_{b} \sum_{i, j}\left[H_{i}^{I} A_{i}\left[H_{j}^{I} A_{j}, \boldsymbol{\rho}\right]\right]
$$

in which $T_{b}$ means tracing over the baby universe sector operators. Assuming then [2] convenient orthonormalization relation and commutation relations for hermitian operators, so as the usual Fock expansion for the quantum field operators in the continuity limit

$\sum_{i, j} \rightarrow \int \mathrm{d}^{4} x_{0}$, after integrating over the insertion points of the baby universes on the large universal spacetime and the momentum that appears in the delta function of the orthonormalization relations using the customary quantum-optical measure [2], we obtain the master equation for the reduced density matrix for matrix elements in the Fock space of the matter field number states in the diagonal representation

$$
\dot{\bar{P}}_{n}(k, t)=-8\left(n+\frac{1}{2}\right)^{2}\left(N+\frac{1}{2}\right) \sinh \left(2 k_{0}\right) \bar{P}_{n}(k, t),
$$

where $N=0,2,4, \cdots$ denotes the initial number of baby universes, $\sqrt{2 k}$ is the proper distance on the wormhole inner 3-manifold between the two correlated points at which two baby universes are simultaneously created or annihilated, and $k_{0}=\sqrt{2 k /\left(R_{0}^{2}-k\right)}$, and we have taken the limit $k \rightarrow 0$, substracting it from the previously derived expression, in order to eliminate changes in the reduced density matrix which do not originate from quantum non-locality. The crucial point for the baby universe pairs now is that the density matrix in scalar particle number representation actually corresponds to a quantum state in position representation whose evolution satisfies a generalized master equation given by [4]

$$
\dot{\bar{P}}_{n}\left(x, x^{\prime}, t\right)=-P\left(n+\frac{1}{2}\right)^{2} \bar{P}\left(x, x^{\prime}, t\right),
$$

with

$$
P=8\left(N+\frac{1}{2}\right) \sinh \sqrt{\frac{4\left(x-x^{\prime}\right)^{2}}{r_{o}^{2}}},
$$

where $r_{0}$ is the radius of the Euclidean wormhole throat and the $\left(x, x^{\prime}\right)$ 's describe the insertion points of the correlated pairs in the otherwise independent universes.

Now, depending on the physical situation we are dealing with, we can have the following two essentially distinct cases. For large objects which are placed as parts (not the whole) of a given universe, a coherent superpo- sition of two Gaussians separated by a space-like distance $\Delta x=x-x^{\prime}$ should be considered (such as Zurek first did [1]). Then, at macroscopic distances, $\Delta x=x-x^{\prime}$ will be much larger than the Gaussian width, and the density matrix will show essentially four distinct peaks, two corresponding to the diagonal elements basically surviving intact the wave packet collapse, and two associated with the off-diagonal elements amounting to the quantum correlations that nearly fully disappear during measurement, giving thus rise to position as an exactly preferred basis. That is a typical example of what is known as the decoherence process leading to classical behaviour of the macroscopic objects that do not entail the whole universe.

In case that you consider a set of universes related to each other by means of e.g. Euclidean and Lorentzian wormholes, all entities pertaining to the very nature of the space-time separated structures, it will be needed that in order for keeping the space-times of the single universes fully independent from each other, one should integrate off the generalized above master equation over the space-like separation $\Delta x=x-x^{\prime}$ in such a way that the final form of that generalized master equation does not explicitly contain any dependence on $\Delta x=x-x^{\prime}$, being this replaced for a necessarily nonzero footprint factor resulting from the integration process. This is the key point which clearly avoids having in the case of a single universe in the multiverse the position as an exactly preferred basis, and hence an available classical result is no longer allowed. Actually, because by the very definition of cosmic space-time, well-defined mutual position between single universes in the multiverse can by no means be established, before any other alternative interpretation might be made, one should be aware that the off-diagonal matrix elements describing quantum correlations between insertion points responsible for the quantum nature of every single universe can by no means be damped, always leaving a definitively nonzero (quantum) footprint factor coming from the double integration over $\Delta x=x-x^{\prime}$ which should be performed both over $\bar{P}$ and $P$ in the generalized master equation, which can no longer be erased off. These memories from the off-diagonal matrix components can no longer be eliminated during any measurement processes (such as it could be made with the two peaks associated with the Zurek off-diagonal elements amounting to the quantum correlations that nearly fully disappear during measurement, giving rise to position as an exactly preferred basis) and, therefore, the decoherence scenario can by no means be driven to its classical completion, so leaving an unavoidably quantum single universe evolving in the sea of all other single universes. 


\section{BABY UNIVERSES AND THE CURRENT ACCELERATING UNIVERSE}

Moreover, there still is another technical reason amounting to enhance even further the conclusion drawn in the previous section in the case of an accelerating universe. In fact, it has been recently shown [5] that the action integral for all kinds of baby universes can be seen to be the same as that for their T-duality symmetric space-times which turn out to be those of accelerating universes. It follows that the accelerating universe sector and the baby universe sector are equivalent physically to each other. Now, the violation of Bell's inequalities which points to the essential quantum nature of the system inducing it is attained when the following quantum-optical inequality holds [6]

$$
C \equiv \frac{\left\langle a^{+} a a^{+} a\right\rangle}{\left\langle a^{+} a a^{+} a\right\rangle+\left\langle\left(a^{+}\right)^{2}(a)^{2}\right\rangle} \geq 0.707
$$

where the $a$ 's are Fock annihilation and creation quantum operators for the given field.

Then, from inequality (5) and the definition of the second-order coherent function,

$$
g_{n}^{(2)}=\frac{\left\langle n^{2}\right\rangle-\langle n\rangle}{\left\langle n^{2}\right\rangle} \geq 1-\frac{1}{\langle n\rangle},
$$

where use has been made of the general condition $\left\langle n^{2}\right\rangle /\langle n\rangle \geq 1$, we get

$$
C=\frac{1}{1+\frac{\langle n\rangle^{2}}{\left\langle n^{2}\right\rangle} g_{n}^{(2)}} \geq \frac{1}{1+g_{n}^{(2)}}
$$

We again compute then the master equation for the second-order correlation function from the matrix elements in the baby universe Fock space of the matter field number states [2], in the diagonal representation, following the same procedure as for obtaining Eq.2, but without expliciting in this case the quantity $k$ in terms of the insertion spatial points, that is

$$
\dot{\bar{P}}_{n}(k, t)=-8\left(n+\frac{1}{2}\right)^{2}\left(N+\frac{1}{2}\right) \sinh \left(2 k_{0}\right) \bar{P}_{n}(k, t),
$$

in which again $N=0,2,4, \cdots$ denotes the initial number of baby universes whenever these are branched off in correlated pairs, $\sqrt{2 k}$ is the corresponding proper distance on the Euclidean wormhole inner 3-manifold between the two correlated points at which two baby universes are simultaneously created or annihilated in this case, and $k_{0}=\sqrt{2 k /\left(R_{0}^{2}-k\right)}$, with $R_{0}$ the smallest value of the scale factor in the connected manifold. Following then the procedure described in Reference [2] we can then derive the evolution rate of the second-order coherence function, to obtain [2]

$$
\begin{aligned}
& \dot{g}_{n}^{(2)}=P\left(N, k_{0}\right) \\
& \cdot\left[\frac{1}{4}\langle n\rangle g_{n}^{(2) 2}+\frac{1}{4}\left(8\langle n\rangle^{2} g_{n}^{(3)}-7\right) g_{n}^{(2)}-\langle n\rangle\left(\langle n\rangle g_{n}^{(4)} 6 g_{n}^{(3)}\right)\right],
\end{aligned}
$$

where $g_{n}^{(3)}$ and $g_{n}^{(4)}$ are the third- and fourth-order coherence functions, respectively, and $P\left(N, k_{0}\right)=8\left(N+\frac{1}{2}\right) \sinh \left(2 k_{0}\right)$. For the vacuum case, Eq.9 admits the exact solution

$$
\begin{array}{r}
g_{0}^{(2)}\left(0, k_{0}\right)=\exp \left(-\frac{7}{2} P\left(N, k_{0}\right) t\right), \text { so that } \\
C \geq \frac{1}{1+\mathrm{e}^{-\frac{7}{2} P\left(0, k_{0}\right) t}} .
\end{array}
$$

Thus, for the vacuum case there will always be a large enough time for which Bell's inequalities are violated. Such a time is smaller than or as most equal to $t_{v}=2 \ln 2.37 /\left(7 P\left(0, k_{0}\right)\right)$. This conclusion is still valid for small, nonzero values of $\langle n\rangle$ and even in the limit of large $\langle n\rangle$ where

$$
g_{n}^{(2)} \cong \frac{1}{2}\left(1+\exp \left[2 P\left(N, k_{0}\right) t\right]\right) .
$$

It finally follows that, though in his limit the right hand side of expression (10) is in this case defined to be smaller than 0.707 even for $t=0$, the inequality relating it with $C$ can leave still a residual room for the violation of Bell's inequalities in the situation where we usually expect the classical limit to hold, so allowing any multiverse descriptions to admit a joint quantum treatment. The conclusion can then be drawn that single accelerating universes unavoidably entail the phenomena of quantum entanglement.

\section{OBSERVERS ARE ALL INSIDE THE UNIVERSE}

Besides the above rather technical reasons, one still may have fundamental theoretical and almost philosophical problems which concern the feature that quantum theory generally splits the world into two parts: the system under study and the rest of the world, which inexorably must contain the observer. Now, cosmology suffers from the paradoxical requirement that no observer can be placed outside the universe. It is in this sense that the universe is therefore doomed to spend eternity like nothing more than a vague possibility, so far from actual 
reality like the Schrödinger's cat. And then quantum cosmological models dictate that therefore we cannot talk about the universe as a whole, but only what a given observer inside it might measure.

Nevertheless, if we keep ourselves within the context of what has been said above, then a quantum-cosmological observer must by itself be split into two unavoidably independent parts: the bone and flesh structures supporting the set of tools made of detectors, food and breathing endorsements and displacement procedures, so as the thermodynamic, psychological and cosmic arrow of times, etc., which all are classical in their very nature and may be really separated from the very concept of quantum observer to join the rest of the world, so leaving the other, now quantum-mechanically relevant part of the quantum observer: its mind. This is the actual and unique designer of every observation or measurement actions or decision making, and clearly should form part of the entangled quantum universe, just playing the role of that completing missing part required by that universe to achieve quantum completion.

\section{CONCLUSIONS AND FURTHER COMMENTS}

As one conclusion we shall notice that the above arguments lend support to the idea that we are daring to suggest in this report that every single universe, in the multiverse or not, is by itself an essentially quantum-mechanical system endowed with all tools of quantum information, which violated Bell's inequalities [7], and could by no means have any classical analog. At first sight it might also be reconsidered that to deal with a macroscopic mass using quantum mechanics one must consequently correct the derivation of the equations describing the evolution of the universe and the behavior of that mass itself. However, besides the feature that the mass density is what matter here and this mass density is extremely small for our currently accelerating universe, the development that we have considered here has noting to do with such an issue but with the nucleation/annihilation balance of those baby universes being branched off and in throughout the large spacetime of the current universe. In fact, as important outcomes from such a balance we have the fixing of all particle masses and physical constants, according an improved Coleman mechanism $[8,9]$ and the existence of quantum-optical master equations as those given by Eqs.3 and 8, according to the mechanisms described in References [2] and [4] for baby universes created in self-correlated pairs. Now, since there will always exist a nonzero contribution for nucleation of pairs of baby universes it follows that the Coleman mechanism for the big fix [8] becomes consistent $[9,10]$. We finally point out that the quan- tum-mechanical entanglement energy for a single universe should be interpreted in terms of the existence of a future event horizon, which is granted for any model of the current accelerating universe, where entanglement is established between the two independent spacetime regions created by that horizon [11].

No boubt, this paper is too brief to provide definitive description, analysis or discussion on the problem of why the universe as a whole ought to be regarded as a quantum object devoid of any classical analog. Even though one therefore cannot provide with a fully consistent answer to the question posed in the title, the paper still contains the main basic ingredients needed to properly cook such an answer which should in any event given in terms of the entangled baby universe pairs.

\section{ACKNOWLEDGEMENTS}

The author thanks Carmen Sigüenza and Salvador Robles-Pérez for enlightening discussions and decisive technical help, and the Estación Ecológica de Biocosmogía de Medellín, Spain, where part of this work was carried out. This paper was supported by MICINN under Research Project No. FIS2008-06332.

\section{REFERENCES}

[1] Zurek, W.H. (1991) Decoherence and the transition from quantum to classical. Physics Today, 36.

[2] González-Díaz, P.F. (1992) Non Classical States in Quantum Gravity. Physics Letters, 293, 294-298. doi:10.1016/0370-2693(92)90886-9

[3] González-Díaz, P.F. (1992) Regaining quantum incohence for matter fields. Physical Review, D45, 499506. doi:10.1103/PhysRevD.45.499

[4] González-Díaz, P.F. (1998) Quantum gravity and the problem of measurement. International Journal of Theoretical Physics, 37, 249-256. doi:10.1023/A:1026610616692

[5] González-Díaz, P.F. and Robles-Pérez, S. (2009) Entangled accelerating universe. Physics Letters B, 679, 298304. doi:10.1016/j.physletb.2009.08.006

[6] Reid, M.D. and Walls, D.F. (1986) Violations of classical inequalities in quantum optics. Physical Review A, 34, 1260-1276. doi:10.1103/PhysRevA.34.1260

[7] Bell, J.S. (1987) Speakable and unspekable in quantum mechanics. Cambridge Univ. Press, Cambridge.

[8] Coleman, S.R. (1988) Why there is nothing rather than something: A theory of cosmological constant. Nuclear Physics B, 310, 643-668.

[9] González-Díaz, P.F. (2011) Is there a bigger fix in the multiverse? e-Print: arXiv: 1102.2771v1.

[10] González-Díaz, P.F. (1993) Blackbody distribution for wormholes. Classical and Quantum Gravity, 10, 2505. doi:10.1088/0264-9381/10/12/009

[11] Müller, R. and Lousto, C.O. (1995) Entanglement entropy in curved spacetimes with event horizons. Physical Review D, 52, 4512-4517. doi:10.1103/PhysRevD.52.4512 